\title{
Cultural Awareness and Sensitivity of Students in a Physical Therapy Program - A Pilot Survey
}

Olaide Oluwole-Sangoseni

Maryville University of Saint Louis, osangoseni@maryville.edu

Michelle Jenkins-Unterberg

Maryville University of St. Louis, munterberg@maryville.edu

Follow this and additional works at: https://nsuworks.nova.edu/ijahsp

Part of the Medicine and Health Sciences Commons

\section{Recommended Citation}

Oluwole-Sangoseni O, Jenkins-Unterberg M. Cultural Awareness and Sensitivity of Students in a Physical Therapy Program - A Pilot Survey. The Internet Journal of Allied Health Sciences and Practice. 2018 Jan 01;16(1), Article 1.

This Manuscript is brought to you for free and open access by the College of Health Care Sciences at NSUWorks. It has been accepted for inclusion in Internet Journal of Allied Health Sciences and Practice by an authorized editor of NSUWorks. For more information, please contact nsuworks@nova.edu. 


\title{
Cultural Awareness and Sensitivity of Students in a Physical Therapy Program - A Pilot Survey
}

\begin{abstract}
Background: Attempts to address health and health care disparities in the United States have led to a renewed focus on the training of healthcare professionals including physical therapists. Current health care policies emphasize culturally competent care as a means of promoting equity in care delivery by health care professionals. Experts agree that cultural insensitivity has a negative association with health professionals' ability to provide quality care.

Objective: To evaluate the cultural awareness and sensitivity of physical therapy (PT) students in a didactic curriculum aimed to increase cultural awareness.

Methods: Using the Multicultural Sensitivity Scale (MSS), a cross-sectional survey was conducted to assess cultural sensitivity among three groups of students, $(\mathrm{N}=139)$ from a doctor of physical therapy (DPT) program at a liberal arts university in Saint Louis, MO.

Results: Response rate was 76.3\%. Participants $(n=100)$ were students in first (DPT1, $n=36)$, third (DPT3, $n=36$ ), and sixth (DPT6, $n=28$ ) year of the program. Mean ranked MSS score was DPT1 $=45.53$, DPT3 = 46.60 DPT6 $=61.91$. Kruskal-Wallis analysis of the mean ranked scores showed a significant difference among three groups, $\mathrm{H}=6.05(2, \mathrm{~N}=100), \mathrm{p} \leq .05$.

Discussion: Students who have completed the cultural awareness curriculum, and undergone clinical experiences rated themselves higher on the cultural sensitivity/awareness. Results provide initial evidence that experiential learning opportunities may help PT students to more effectively integrate knowledge from classroom activities designed to facilitate cultural competence.
\end{abstract}

\section{Author Bio(s)}

Dr. Olaide Oluwole-Sangoseni, PT, PhD, DPT, MSc,GCS is an Assistant Professor of physical therapy at Maryville University of Saint Louis, Missouri. She is also a licensed physical therapist in the state of Missouri.

Dr. Michelle Unterberg, PT, DHS, is an Associate Professor of physical therapy and Assistant Dean of College of Health Sciences at Maryville University of Saint Louis, Missouri. She is also a licensed physical therapist in the state of Missouri.

\section{Acknowledgements}

We acknowledge Sandy Ross, PT, DPT, MHS, PCS for her review contributions and the physical therapy students at Maryville University of Saint Louis for their participation. 


\title{
TIAHSP \\ The Internet Joumnal of Allied Health Sciences and Practice \\ Dedicated to allied health professional practice and education
}

Vol. 15 No. 4 ISSN 1540-580X

\section{Cultural Awareness and Sensitivity of Students in a Physical Therapy Program - A Pilot Survey}

\author{
Olaide Oluwole-Sangoseni, PhD, DPT, MSc, GCS \\ Michelle Jenkins-Unterberg, PT, DHS \\ Maryville University of Saint Louis
}

United States

\begin{abstract}
Background: Attempts to address health and health care disparities in the United States have led to a renewed focus on the training of healthcare professionals including physical therapists. Current health care policies emphasize culturally competent care as a means of promoting equity in care delivery by health care professionals. Experts agree that cultural insensitivity has a negative association with health professionals' ability to provide quality care. Objective: To evaluate the cultural awareness and sensitivity of physical therapy (PT) students in a didactic curriculum aimed to increase cultural awareness. Methods: Using the Multicultural Sensitivity Scale (MSS), a cross-sectional survey was conducted to assess cultural sensitivity among three groups of students, $(\mathrm{N}=139)$ from a doctor of physical therapy (DPT) program at a liberal arts university in Saint Louis, MO. Results: Response rate was 76.3\%. Participants ( $n=100$ ) were students in first (DPT1, $n=36$ ), third (DPT3, $n=36$ ), and sixth (DPT6, $n=28$ ) year of the program. Mean ranked MSS score was DPT1 $=45.53$, DPT3 $=46.60$, DPT6 $=61.91$. Kruskal-Wallis analysis of the mean ranked scores showed a significant difference among three groups, $\mathrm{H}=6.05(2, \mathrm{~N}=100), \mathrm{p} \leq .05$. Discussion: Students who have completed the cultural awareness curriculum and have undergone clinical experiences rated themselves higher on the cultural sensitivity/awareness. Results provide initial evidence that experiential learning opportunities may help PT students to more effectively integrate knowledge from classroom activities designed to facilitate cultural competence.
\end{abstract}

\section{INTRODUCTION}

Health policy experts and organizational studies on healthcare disparities have identified cultural incompetence of health care professionals as a contributing factor to inaccessibility to care, reduced care seeking behavior, and dismal outcome of care in developed societies. ${ }^{1-4}$ In the United States, data showed that minority populations are the most affected by health disparities. ${ }^{1-}$ ${ }^{5}$ Healthcare policies emphasize culturally competent care to promote equity in care delivery because negative associations have been observed between decreased cultural awareness and insensitivity and perceived quality of care delivered. ${ }^{4-10}$ Thus, addressing healthcare inequities or disparities has led to a renewed focus of health policy experts and researchers on cultural competence training of healthcare professionals including physical therapists. ${ }^{4-16}$ The American Physical Therapy Association supports the provision of culturally congruent care; it recognizes cultural competence as a continuous process rather than an end. The Association's patient-centered vision statement approved by the House of Delegates in 2013 states, "The physical therapy profession embraces cultural competence as a necessary skill to ensure best practice in providing physical therapy services by responding to individual and cultural considerations, needs, and values."10

Cultural competence is a multidimensional construct that includes cultural awareness, cultural attitude, cultural skill, and multicultural or cross-cultural experiences. ${ }^{1-6}$ Culture is a shared set of beliefs, values, and morals that reflect an individual's worldview and way of life..$^{3-6}$ Culture may be shared by ethnic groups. Ethnicity is an expression of cultural distinctiveness in political and socioeconomic situations. $3,5,7,8$ That is, an individual's ethnic identity may determine their culture. Cultural sensitivity is a component of cultural awareness, which in turn is a construct of cultural competence. ${ }^{2,3,5}$ Cultural awareness, the first step toward cultural competence and the focus of this study, is the ability of healthcare practitioners to recognize, value, and consider their patient's or client's cultural worldview. ${ }^{13,17}$ Self-awareness, an essential part of cultural awareness, allows the healthcare practitioners to self-reflect and understand their own implicit bias during care delivery. There are many theoretical frameworks

(C) The Internet Journal of Allied Health Sciences and Practice, 2017 
to the understanding of the concept of cultural competence including awareness of oneself and others, knowledge of other cultures, and a non-judgmental ability to meet specific cultural needs. . $, 3,5,7,16$

Maryville University of St. Louis in Missouri has developed and implemented various academic courses, programs, and campus activities to increase students' cross-cultural awareness. In addition, in an effort to meet program specific accreditation requirements, the College of Health Professions and the physical therapy (PT) program have gone further to integrate cultural competency studies in a spiraling manner (step-wise method of integrating the framework of cultural competence with increasing complexity while reinforcing previous learning ${ }^{18}$ ) in the didactic curriculum at the undergraduate education phase and during doctoral studies before and after clinical rotations. The PT program is a 6.5-year, freshman-entry doctoral education program. As a freshman-entry program, all students follow an established curriculum that includes a foundational course in cultural diversity to introduce and teach applicable cultural concepts. As the students' progress in the program, the concept is expanded and interwoven into multiple courses. This model is guided by the recommendation of the National Center for Cultural Competence that the most effective approach to cultural competency training should be a benchmark individual inventory or self- assessment on cultural sensitivity and awareness followed by a tailored educational curriculum that imparts knowledge and allows practice. ${ }^{1,11}$

Given the fact that available research findings ${ }^{4,5,7,8,8,13}$ are mixed regarding the most effective method of teaching cultural competence, this study aimed to evaluate the impact of an academic curriculum on cultural perceptions of doctor of physical therapy (DPT) students in a 6.5 years' program. In this pilot investigation, the goal of this study was to examine the impact of a dedicated didactic curriculum with purposeful attention to promoting cultural sensitivity and awareness. The purpose of this study was to determine how students' cultural self-perceptions, attitudes, and cultural awareness ratings differ at different levels of the DPT program.

\section{METHODOLOGY}

The study was approved by the Institutional Review Board at Maryville University of St. Louis.

\section{Participants}

Midway through the 16-week fall semester of the academic year at Maryville University of Saint Louis, a private university located in the midwest of the United States, three cohorts of students were invited to participate in a survey about cultural perceptions of themselves and others. Students were in their first (DPT1), third (DPT3) and sixth (DPT6) years of the freshman-entry DPT program. A freshman-entry physical therapy program means that from the first year, students in the undergraduate program (1st, $2^{\text {nd }}, 3^{\text {rd }}$ years $)$ have guaranteed admission into the graduate phase $\left(4^{\text {th }}, 5^{\text {th }}\right.$, and $6^{\text {th }}$ years of the DPT program once they complete and earn the baccalaureate degree prerequisites. Thus, first and third-year students in this study were in the pre-clinical phase of the program; the sixth year students were in the clinical phase. All participants were contacted via their University issued students' email accounts on record (Figure 1). Participants were invited to complete the one-time survey anonymously, which required up to 10 minutes. They were informed that they qualified for the study because of their enrollment in the DPT program. In order to increase the likelihood of participation, the initial email invitation included a brief explanation of the purpose of the study and a link to access the actual survey. Email follow-up reminders were sent once weekly for four weeks. A final reminder was sent in the final week. The survey was open for six weeks. 


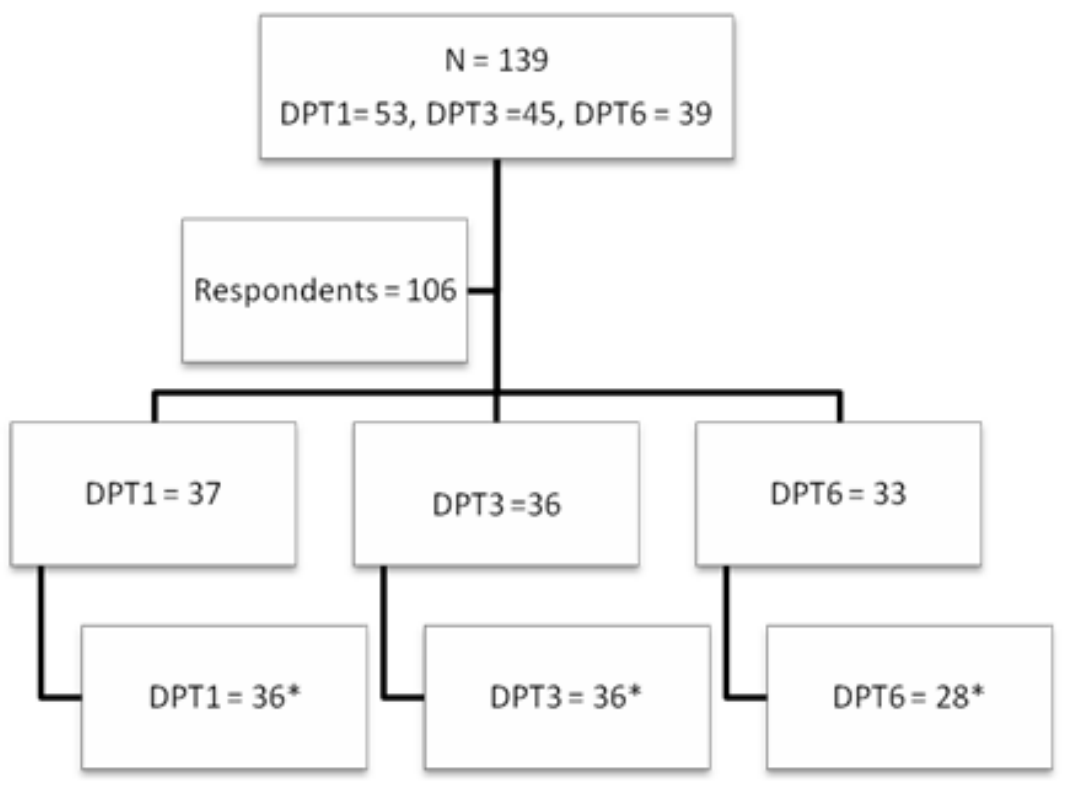

${ }^{*}$ Respondents who completed $\geq 75 \%$ of survey items including demographic information.

Figure 1: Flow Chart of Survey Respondents

Description of each group of participants' prior learning is provided (Figure 2). All first-year students are expected to participate in a weeklong annual cultural advocacy immersion program, which is a university-wide diversity and inclusion advocacy event created by volunteer members of the university community including students, staff, and faculty. The event features walk-through poster displays and short videos on issues such as oppression, privilege, and power. Students are invited to view information displays on issues of xenophobia, racism, heterosexism, and classism. This annual event is advertised through all student organizations. Faculty members are urged to encourage students in their classes to voluntarily participate by addressing elements of the event as applicable in their courses. According to J. McCluskey, PhD, the vice president of the office of First Year-Experience, internal tracking data showed a participation rate of approximately $100 \%$ by all 400 first year students regardless of their academic major. ${ }^{19}$ 


\begin{tabular}{|c|c|c|c|c|c|c|c|c|c|c|c|}
\hline \multicolumn{2}{|l|}{ Year 1} & \multicolumn{2}{|l|}{ Year 2} & \multicolumn{2}{|l|}{ Year 3} & Year 4 & & \multicolumn{2}{|l|}{ Year 5} & \multicolumn{2}{|l|}{ Year 6} \\
\hline \multicolumn{6}{|c|}{ Pre-professional phase } & \multicolumn{6}{|c|}{ Professional phase } \\
\hline $\begin{array}{l}\text { Semester } \\
1^{*}\end{array}$ & $\begin{array}{l}\text { Semester } \\
2\end{array}$ & $\begin{array}{l}\text { Semester } \\
3\end{array}$ & $\begin{array}{l}\text { Semester } \\
4\end{array}$ & $\begin{array}{l}\text { Semester } \\
5^{*}\end{array}$ & $\begin{array}{l}\text { Semester } \\
6\end{array}$ & $\begin{array}{l}\text { Semester } \\
7\end{array}$ & $\begin{array}{l}\text { Semester } \\
8\end{array}$ & $\begin{array}{l}\text { Semester } \\
9\end{array}$ & $\begin{array}{l}\text { Semester } \\
10\end{array}$ & $\begin{array}{l}\text { Semester } \\
11^{*}\end{array}$ & \begin{tabular}{|l} 
Semester \\
$12^{\star \star}$ \\
\end{tabular} \\
\hline \multicolumn{12}{|c|}{$\begin{array}{l}\text { 1week Tunnel of Consciousness live event attendance- a weeklong university wide cultural advocacy immersion program to expose students to } \\
\text { social justice issues and diversity awareness project. }\end{array}$} \\
\hline & & & \multicolumn{9}{|c|}{$\begin{array}{l}16 \text { weeks Didactic Course: Cultural Diversity in Health and Illness- emphasize cultural competency in } \\
\text { interpersonal relationships, within and outside the clinical environment. Students recognize what it means to } \\
\text { be multicultural, and how culture may influence healthcare behaviors. }\end{array}$} \\
\hline & & & & \multicolumn{8}{|c|}{$\begin{array}{l}\text { 16weeks Didactic Course: Health care Ethics - Students understand that factors that influence } \\
\text { health disparities are complex; how practitioner's ethical or unethical behavior may have a } \\
\text { strong impact of patient's outcomes. }\end{array}$} \\
\hline & & & & & & & & \multicolumn{4}{|c|}{$\begin{array}{l}\text { 10weeks Clinical Experience - Students } \\
\text { understand the importance of cultural } \\
\text { competence in the delivery of care; designing } \\
\text { appropriate plan of care and communicate } \\
\text { goals to patients in cross-culturally effective } \\
\text { wavs to ensure ootimal outcome. }\end{array}$} \\
\hline & & & & & & & & & & \multicolumn{2}{|c|}{$\begin{array}{l}\text { 16weeks Didactic } \\
\text { Course: Management } \\
\text { of Ethical Physical } \\
\text { Therabv Practice }\end{array}$} \\
\hline
\end{tabular}

*Survey completed midway through the fall semester.

${ }^{* *}$ Semester 12 - Year 6 is the final semester with some on-campus didactic classes; Semester 13 - Year 7 is clinical experiences at various types of practice settings in the United States.

Figure 2: Participants prior learning experiences

In the spring of their second year, the surveyed DPT3 students completed a 15 contact hour didactic course titled, 'Cultural Diversity in Health and IIIness' and participated in the same cultural advocacy immersion event in the fall semester of their third year. This course explored the theoretical and conceptual aspects of cultural healthcare. Through a combination of lectures, discussions, role-play, and group work, topics explored included culture and ethnicity, cultural variations in response to actual or potential problems of health and illness, and research literature reviews on methods of caring for and treating individuals with culturally influenced responses. At the time of the survey, students were enrolled in a 15 contact hours didactic course titled, 'Health care Ethics.' Instructional activities included classroom lectures, discussion, case studies, and role-playing of crosscultural scenarios that may have ethical implications. The course focused on the exploration of ethical theories relevant to the development of the ethical dimension of the students' growth as competent and caring healthcare professionals.

Students in the sixth year completed these two courses in addition to all other academic courses in preparation for real-life clinical interactions. At the time of the study, these students had completed two, five-week clinical experiences in the previous summer semester. These experiences allowed them to synthesize the lessons from theoretical frameworks outlined in their previous didactic courses and apply it to real-life clinical situations. At the time of the survey, the DPT6 students were enrolled in multiple 15 contact hour physical therapy clinical courses, which included didactic classroom work and hands-on, on-campus laboratory experiences, which may have included interactions with volunteer members of the community in preparation for their three, ten-week clinical experiences.

Upon providing Informed consent, each voluntary participant was invited to access an online version of the Multicultural Sensitivity Scale (MSS) ${ }^{9}$ available through university licensed software (Qualtrics $\left.{ }^{\circledR}\right)$. All participants were directed to complete the survey based on cultural perceptions of themselves and others. 
The Multicultural Sensitivity Scale

The 21-item MSS used in this study measured attitudes toward race, ethnic identity, cultural tolerance, and sense of belonging..$^{9,20}$ The MSS is an open-access survey that has been validated. ${ }^{9,20}$ The MSS was initially developed from a synthesis of cross-cultural experiences directed toward the assessment of secondary school health educators and nurses. ${ }^{20}$ The developer's pilot study supported its reliability and validity; it had an internal consistency of 0.90 among school health educators $(n=31)$, and 0.89 in a sample of nurses $(n=150) .{ }^{20}$ It has been validated and used in a limited number of studies involving students in a physician assistant program, a nutrition program, and a school-based nursing program.9,12,14,15 This tool was published for open-access use for health professions students by the Center for International Rehabilitation Research Information and Exchange in alliance with the National Institute of Disability, Independent Living, and Rehabilitation Research. ${ }^{21}$ However, the MSS has not been used in physical therapy literature. Four demographic items (age range, gender, race, and class year) were added to the online survey to be able to provide specific characteristics of our study population.

Item response total score (TS) was computed for each participant. The TS was the sum of the MSS items. ${ }^{9}$ The possible score range was 21 to 135 points; there are no specific cut-off scores for levels of cultural awareness. Responses on the 6-point Likert scale were initially dichotomized to 1) agree for values 'slightly agree,' 'agree,' and 'strongly agree', and 2) disagree for values 'slightly disagree,' 'disagree,' and 'strongly disagree' responses between the three DPT classes to determine between group differences. Based on the wording of the questions, a higher score in this study was indicative of a higher level of cultural awareness or sensitivity. Sum mean and median score differences among groups were analyzed using Kruskal-Wallis test at $p$ $\leq 0.05$. The frequency of the responses was checked for similarity in distributions needed to meet the statistical assumption for the Kruskal-Wallis test. Post hoc analysis using Fishers Least Significant Difference (LSD) test was conducted to determine wherein the difference laid among DPT classes. The choice of LSD was based on our statistician recommendation that the LSD test computes the pooled standard deviation from all the groups, therefore, increases the power to prevent false positives.

\section{RESULTS}

Survey response rate was $76.3 \%$ (106/139); 6 people did not provide demographic information, which invalidated their responses. Demographic distribution for each group is shown (Table 1). Groups' demographic characteristics were similar enough for the observed differences to be ascribed to years of training.

Table 1. Characteristics of Participants

\begin{tabular}{|l|c|c|c|}
\hline Demographic Variable & Year 1 & Year 3 & Year 6 \\
\hline Available Student Population (139) & 53 & 45 & 39 \\
\hline Response Rate (\%) & 65.5 & 80.0 & 71.8 \\
\hline & $\mathrm{n}=36$ & $\mathrm{n}=36$ & $\mathrm{n}=28$ \\
\hline Females & 29 & 27 & 21 \\
\hline Age Range (years) & 18 to 20 & 21 to 23 & 24 to 27 \\
\hline Race & 32 & 31 & 27 \\
Non-Hispanic White & 0 & 1 & 0 \\
Hispanic & 0 & 0 & 0 \\
Black/African-American & 1 & 0 & 0 \\
Asian & 0 & 0 & 0 \\
Native American & 0 & 0 & 0 \\
Pacific Islander & 1 & 1 & 1 \\
Other & 3 & 3 & 0 \\
No information & & & \\
\hline
\end{tabular}

The percentage rates of agreement and disagreement among participants are presented (Appendix 1). Median scores (Table 2) and total scores for items related to ethnic identity were highest for the sixth year students. For example, items $1,7,15$, and 16 received a sum score of 132, 135, 119, and 121 for DPT6 compared to 119, 110, 106, and 101 for DPT1, and 127, 129, 111, and 97 for DPT3, respectively (Appendix 2). The frequency distribution of the responses was checked for variability and the score distributions among each group were found to be sufficiently bell-shaped to justify the use of the Kruskal-Wallis and Fishers LSD test for post hoc analysis. That is, the scores for each group have the same shape and variability. The analysis (Table 2) showed there was a weak significant difference among mean ranked group scores, $H=6.05(2, N=100), p \leq 0.05$. However, post hoc analysis did not categorically show how the groups differ significantly from each other (Table 3 ).

(C) The Internet Journal of Allied Health Sciences and Practice, 2017 
Table 2. Comparison of ranked total scores among DPT classes

\begin{tabular}{|c|c|c|c|c|c|}
\hline \multicolumn{5}{|c|}{ Total Score Ranks } & \\
\hline DPT class & $N$ & Median & Mean Rank & $\begin{array}{c}\text { Test } \\
\text { Statistics }{ }^{a, b}\end{array}$ & \\
\hline DPT1 & 36 & 55.0 & 45.5 & & \\
\hline DPT3 & 36 & 57.0 & 46.6 & & Total Score \\
\hline DPT6 & 28 & 61.5 & 61.9 & Chi-Square & 6.049 \\
\hline Total & 100 & & & $\mathrm{df}$ & 2 \\
\hline & & & & Asymp. Sig. & 0.049 \\
\hline
\end{tabular}

a. Kruskal Wallis Test

b. Grouping Variable: DPT class

\begin{tabular}{|c|c|c|c|c|c|c|}
\hline \multirow[t]{2}{*}{ (I) DPT class } & \multirow[t]{2}{*}{ (J) DPT class } & \multirow{2}{*}{$\begin{array}{c}\text { Mean Difference } \\
(I-J)\end{array}$} & \multirow{2}{*}{$\begin{array}{l}\text { Std. Error } \\
( \pm)\end{array}$} & \multirow[t]{2}{*}{$p$-value } & \multicolumn{2}{|c|}{$95 \%$ Confidence Interval } \\
\hline & & & & & Lower Bound & Upper Bound \\
\hline \multirow{2}{*}{ dpt1 } & dpt3 & .47222 & 2.57931 & .855 & -4.6470 & 5.5914 \\
\hline & dpt6 & -4.63889 & 2.75740 & .096 & -10.1116 & 0.8338 \\
\hline \multirow{2}{*}{ dpt3 } & dpt1 & -.47222 & 2.57931 & .855 & -5.5914 & 4.6470 \\
\hline & dpt6 & -5.11111 & 2.75740 & .067 & -10.5838 & 0.3616 \\
\hline \multirow{2}{*}{ dpt6 } & dpt1 & 4.63889 & 2.75740 & .096 & -0.8338 & 10.1116 \\
\hline & dpt3 & 5.11111 & 2.75740 & .067 & -0.3616 & 10.5838 \\
\hline
\end{tabular}

Dependent Variable: Total Score LSD

\section{DISCUSSION}

The pilot study aimed to understand the impact of indirectly structured academic curriculum on cultural perceptions of physical therapy students. The students enroll in only one course with an explicit introduction to the concept of cultural competence, after which the concept of global and multicultural competence training was integrated into other specific courses in the curriculum. The authors sought to determine if the number of years in a spiral educational curriculum with purposeful attention to cultural awareness is effective in promoting cultural sensitivity and awareness. ${ }^{18}$

Students' cultural self-perceptions differed at different levels in the program with an upward trend in cultural awareness gains as student completed clinical phases of the program. The DPT6 cohort had the overall highest total score. However, individual rating of some questions had a higher score for the DPT1 and DPT3 cohorts. We posited the higher rating may be that students in the DPT1 and DPT3 cohorts overestimated their self-perceptions compared to the DPT6 students since the former groups had not completed clinical experience, which may have permitted them to have cultural encounters that challenged their skills. ${ }^{22}$ For example, a higher number of DPT1 and DPT3 students disagreed with statements such as, "I would feel more relaxed if I could work with people of my own ethnic group." This response may be an indication of an overestimation of their own level of self-awareness or it may be that some participants in these groups were providing socially desirable responses. ${ }^{20}$ Another indication of this tendency for social desirability bias was observed in the earlier responses to item 13 that questioned, "In order to be accepted by persons of other ethnic groups, I frequently find myself altering my behavior" (Appendix 1). A majority of the DPT1 and DPT3 groups agreed to this statement. Further, among items related to avoidance of multicultural encounters, there were statistically significant differences between the cultural sensitivity individual item-specific scores of the DPT6 and itemspecific scores of both DPT1 and DPT3 cohorts. For example, first-year students' item-specific scores were lowest for statements such as, "I feel tense and uptight when I have to work closely with persons who are of a different ethnic group than my own," and "The ethnic group that persons belong to frequently determines how I respond to them interpersonally." These responses may be indicative of some level of cultural insensitivity and cultural intolerance. 
Based on the individual item scores, there were high variabilities within each group's responses related to the strength of agreement and disagreement with items. This revealed a higher degree of multicultural sensitivity and tolerance. ${ }^{9}$ For example, comparisons of item specific scores among items $5,6,20$, and 21 related to the concept of tolerance during cross-cultural encounters or interactions showed that a greater proportion of the DPT6 students chose to "strongly disagree" compare to the DPT1 and DPT3 students who were more likely to "slightly disagree" or "disagree" (Appendix 2). A possible explanation for this finding may relate to DPT6 students having greater curricular interventions in the professional years that are designed to facilitate students' application and syntheses of cultural competence concepts or that DPT6 students had a clearer understanding of their own cultural biases and were able to decipher the dynamics of the differences that exist among cultures. The DPT1 and DPT3 cohorts consist of pre-professional students who are still in the early phases of knowledge acquisition, developing and refining their cultural awareness skills. This finding is similar to observations made in previous studies, whereby significant improvement in cultural awareness among healthcare practitioners occurred only when the concepts embedded in the curriculum were put into practice. ${ }^{8,12}$ Similar to findings reported in an earlier study with physicians, DPT6 students may have had more opportunities during clinical experiences to examine and confront privately held racial stereotypes that may influence their clinical decisionmaking process. 4,16

Students' attitudes and cultural awareness ratings also differed at different levels in the program. DPT6 students demonstrated a higher level of awareness of their own implicit and explicit cultural biases and beliefs, but also a significant appreciation of the relationship between their beliefs and cultural differences. These students have been provided with opportunities to integrate prior knowledge from didactic learning about cultural practices into practice through patient interactions. In this study, a proactive approach to increase students' cultural sensitivity and awareness seemed to facilitate students' journeys toward cultural competence. These results showed a seemingly higher level of cultural sensitivity, awareness, and improved attitudes among students who had patient interaction beyond the classroom. The growth and development is expected to translate into their professional lives.

\section{LIMITATIONS}

Multiple limitations in this pilot study warrant cautious interpretation of our findings. This study was purposefully sampled to include different cohorts from the same program from a single educational institution. Second, the self-report nature of the MSS scale may have promoted social desirability bias. Although, the effect of this bias may be small or equal among the groups of participants. Third, the racial homogeneity of the students in this study was high, suggesting little ethnic diversity in the sample population. Fourth, group characteristics were similar enough for the observed differences to be ascribed to years of training; this was a cautious assumption because participants' previous cross-cultural experience was unknown. Prior experience may influence the level of cultural awareness and skills. Finally, statistical power was limited due to the less than expected net sample size $(n=100)$ which may have played a role in the interpretation of the observed significance of some of the statistical comparisons conducted. Post hoc power analysis suggested a minimum sample size of 102 participants was needed to justify the use of parametric tests and to obtain statistical power at the recommended 0.80 . We made an effort to address this by using nonparametric tests for analysis of between-group differences. Despite these limitations, the results of this study demonstrate evidence of the effectiveness of a spiraling curriculum tailored to the promotion of cultural competency among DPT students.

\section{CONCLUSION}

Students who have undergone clinical experience appeared to be more culturally sensitive than pre-clinical students who have only completed the didactic training. Multicultural sensitivity score patterns showed students become more culturally sensitive as they progress through the curriculum and able to apply their knowledge to practice. More research is needed to elucidate the most effective curricula approach to maximize cultural sensitivity training to develop culturally competent clinicians of tomorrow. Further research should include a comparative assessment of outcomes in physical therapy programs with a different curricular approach such as experiential versus non-experiential approach or a short versus long-term focus. Future research should examine the same concept in a larger population of PT students whose demographics closely resemble that of practicing physical therapists. Research should include the use of outcome measures that are not self-reported and examine the translation of cultural competence student training to professional practice.

\section{REFERENCES:}

1. Butler M, McCreedy E, Schwer N, Burgess D, Call K, et al. Improving Cultural Competence to Reduce Health Disparities [Internet]. Rockville (MD): Agency for Healthcare Research and Quality (US); 2016 Mar. [PMID: 27148614] Available at: https://www.ncbi.nlm.nih.gov/pubmedhealth/PMH0087116/. Accessed March 14, 2017.

() The Internet Journal of Allied Health Sciences and Practice, 2017 
2. Agency for Healthcare Research and Quality. CAHPS Cultural Competence Item Set. U.S. Department of Health \& Human Services, June 2012. Available at: https://www.ahrq.gov/cahps/surveys-guidance/item-sets/cultural/index.html Accessed March 14, 2017.

3. National Center for Cultural Competence. The Compelling Need for Cultural and Linguistic competence. Georgetown University Center for Child and Human Development. [Internet]. Available at:

http://nccc.georgetown.edu/foundations/need.html. Accessed March 14, 2017.

4. Chapman EN, Kaatz A, Carnes M. Physicians and Implicit Bias: How Doctors May Unwittingly Perpetuate Health Care Disparities. J Gen Internal Med. 2013;28(11):1504-10. [PMID: 23576243]

5. Betancourt JR, Green AR, Carrillo JE, Ananeh-Firempong $O 2^{\text {nd }}$. Defining cultural competence: a practical framework for addressing racial/ethnic disparities in health and health care. Public Health Rep. 2003;118(4):293-302. [PMID: 12815076]

6. Vaughn LM, Jacquez F, Baker RC. Cultural health attributions, beliefs, and practices: effect on health care and medical education. Open Med Educ J. 2009;2:64-74.

7. Horvat L, Horey D, Romios P, Kis-Rigo J. Cultural competence education for health professionals. Cochrane Database Syst Rev. 2014 May 5;(5):CD009405. doi: 10.1002/14651858.CD009405.pub2. Review. [PMID: 24793445]

8. Beach MC, Price EG, Gary TL, Robinson KA, Gozu A, et al. Cultural competence: a systematic review of health care provider educational interventions. Med Care. 2005;43(4):356-73.

9. Jibaja ML, Sebastian R, Kingery P, Holcomb JD. The multicultural sensitivity of physician assistant students. J Allied Health. 2000;29(2):79-85. [PMID: 10874334]

10. American Physical Therapy Association. Cultural Competence and the Vision. [Internet]. Available at: http://www.apta.org/CulturalCompetence/Vision/. Accessed March 14, 2017.

11. Panzarella KJ. Beginning with the end in mind: evaluating outcomes of cultural competence instruction in a doctor of physical therapy programme. Disabil Rehabil. 2009;31(14):1144-52.

12. Suk MH, Oh WO, Im YJ, Cho HH. Mediating effect of school nurses' self efficacy between multicultural attitude and cultural sensitivity in Korean elementary schools. Asian Nurs Res (Korean Soc Nurs Sci). 2015;9(3):194-9. [PMID: 26412622]

13. Blanchet-Garneau A. Critical reflection in cultural competence development: $A$ framework for undergraduate nursing education. J Nurs Educ. 2016 Mar;55(3):125-32. [PMID:26926212]

14. Schneider V, Thompson BM, Celis MI. Multicultural sensitivity of physician assistant students after instituting cultural competence instruction. Persp Phys Asst Educ. 2005;16(2):103-6.

15. Karpinski $C$, Heinerichs $S$. Exploring the effect a speaker series has on student's level of multicultural sensitivity and cultural competence awareness. Internet J Allied Heal Sci Pract. 2015;13(3):6.

16. Chun MB, Jackson DS, Lin SY, Park ER. A comparison of surgery and family medicine residents' perceptions of crosscultural care training. Hawaii Med J. 2010;69(12):289-93. [PMID: 21225585]

17. Jirwe M, Gerrish K, Emami A. The theoretical framework of cultural competence. J Multicultural Nurs Health. 2006;12(3):616.

18. Harden R, Stamper N. What is a spiral curriculum? Med Teachr. 1999;21(2):141-3. http://dx.doi.org/10.1080/01421599979752

19. McCluskey J. Who are our Students? Lecture presented at: Maryville University of Saint Louis. May 16, 2017; Saint Louis, MO.

20. Jibaja-Rusth ML, Kingery PM, Holcomb JD, Buckner WP, Pruitt BE. Development of a Multicultural Sensitivity Scale. J Heal Educ. 1994;25(6):350-7.

21. Center for International Rehabilitation Research Information and Exchange (CIRRIE) Multicultural Sensitivity Scale. [Internet] Available at: http://cirrie.buffalo.edu/culture/curriculum/activities/scale.php. Accessed March 14, 2017.

22. Campinha-Bacote J. The Process of Cultural Competence in the Delivery of Healthcare Services: a model of care. $J$ Transcult Nurs. 2002 Jul;13(3):181-4. [PMID: 12113146] 